\section{sciendo}

DOI: 10.2478/ncr-2018-0007

(c) Article authors. This is an open access article distributed under the Creative Commons Attribution-NonCommercial-NoDerivs licens. (http://creaticecommons.org/licenses/by.nc-nd/3.0/).

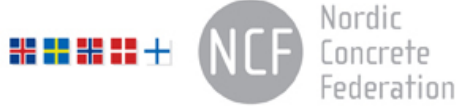

ISSN online 2545-2819

ISSN print $\quad 0800-6377$

Received: Feb. 25, 2018

Revision received: May 27, 2018

Accepted: May 28, 2018

\title{
Filler and Water Reducer Effects on Sedimentation, Bleeding and Zeta-Potential of Cement Paste
}
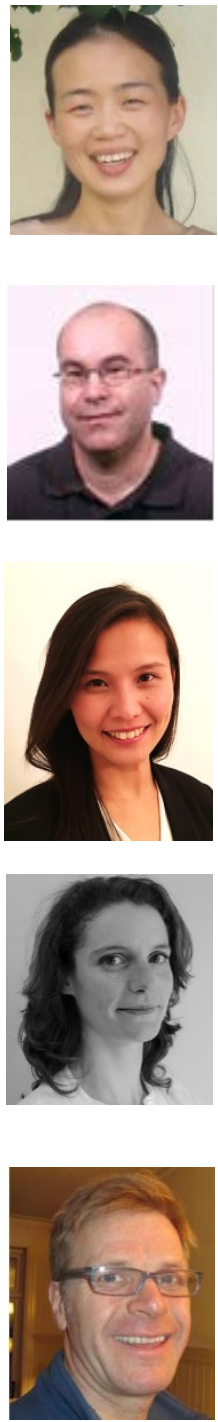

Ya Peng

Post Doc. Researcher

Dept of Structural Eng., NTNU

N-7491Trondheim

yapeng2007@hotmail.no

Bård Pedersen

Senior Principal Engineer

Norwegian Public Roads Administration (NPRA)

$\mathrm{N}-0033$ Oslo

baard.pedersen@vegvesen.no

Serina $\mathrm{Ng}$

Scientist

Building and Infrastructure, Sintef

N-7491 Trondheim

akfc@hotmail.sg

Klaartje de Weerdt

Associate Professor

Dept of Structural Eng., NTNU

N-7491Trondheim

klaartje.d.weerdt@ntnu.no

Stefan Jacobsen

Professor

Dept of Structural Eng., NTNU

N-7491Trondheim

stefan.jacobsen@ntnu.no 


\begin{abstract}
Bleeding and sedimentation quantify the stability of fresh cement paste, whereas the addition of fillers and water reducers affect the stability. The effect of various types of fillers and water reducers was investigated by measuring bleeding, hydrostatic pressure and electroacoustic zetapotential. Depending on their characteristics and use, fillers can improve stability by reducing sedimentation rate and bleeding. The combined effects of fillers and water reducers on the sedimentation rates, quantified as time-dependent hydrostatic pressure changes $(d p / d t)$ in fresh matrix, correspond to their effects on zeta-potentials. The influence of the water reducers on sedimentation and bleeding exceed that of filler type.
\end{abstract}

Key words: stability, sedimentation, bleeding, zeta-potential, filler, water reducer

\title{
1. INTRODUCTION
}

Concrete can generally be regarded as a two phase material with a fluid phase and a particle phase. The properties of a given concrete are determined by the properties of each phase and the proportions between the two phases. Many models have defined the materials in each phase, among which the Particle-Matrix model (PM model) [1] suggests all particles $>125 \mu \mathrm{m}$ as the particle phase and all fluids and particles $\leq 125 \mu \mathrm{m}$ as the matrix phase. Self-compacting concrete (SCC) was developed and is used for its advantage of not needing vibration. Two important properties of SCC are its flowability and its stability. Based on the PM model, the low yield stress and viscosity of the matrix and the density difference between the particles and the fluid causes particles to sink, resulting in sedimentation and bleeding, which are important factors in the instability of SCC [2]. According to Hammer et al. [3], the stability of the matrix itself affects the fresh properties of SCC by contributing to the bleeding and/or inhomogeneity of the concrete. Traditionally, bleeding has been seen as the main form of cement paste and matrix instability. However, sedimentation is also a part of the bleeding process and it has been suggested that the sedimentation rate can be used to evaluate the stability of modern cement matrix with admixtures and mineral additives [4].

For cementitious materials, filler addition is a good way to improve both the flow and stability simultaneously because it increases the matrix volume without adding extra cement [5]. Cement can be replaced by filler to reduce the cost and optimize some fresh properties of concrete such as rheology and stability. However, requirements for strength and durability in terms of $w / c$ or water binder ratio must always be met. Filler can therefore be applied to concrete in two different ways: either to add it to decrease the water to powder ratio (w/p) while keeping $w / c$ unchanged, or to increase $w / c$ by replacing part of cement while keeping w/p constant. Addition and replacement both influence the sedimentation process by changing the solid fraction, the particle size distribution and the total surface area. As the solid fraction $\Phi$ increases or the particle size decreases, the influence of surface forces relative to body forces increases. Flocculation occurs in colloidal mixtures (suspensions consisting of colloid particles with one or more linear dimension between about $1 \mathrm{~nm}$ and $1 \mu \mathrm{m}$ suspended in a fluid [6]). The particles, both the flocs and the single particles, have associated and/or adsorbed ions, molecules and/or liquid pockets so that the volume or thickness of "free" suspending fluid can vary greatly. The addition of admixtures influences the flocculation state of the suspension by electrostatic repulsion and steric hindrance. These interactions affect the effective "particle" sinking velocity during sedimentation. Depending on filler characteristics (particle size, specific surface, flocculation state, etc.) and combination with water reducer, the filler can affect the stability to various degrees. 
Theories including Stokes' Law [7], Richardson-Zaki Equation (R-Z) [8], Kynch theory [9], Kozeny-Carman Equation $(K-C)[10,11]$ etc. describe particle sedimentation and bleeding. As to the experimental method, it has long been recognized that the zeta-potential is a good index of the interaction magnitude between colloidal particles. For colloidal stability, the repulsive forces must be dominant i.e. the greater the zeta-potential the more likely that the suspension is stable. If the zeta-potential is close to zero, the tendency for flocculation increases [12, 13]. Measurements of zeta-potential are commonly used to assess the stability of colloidal systems [14]. On the other hand, non-intrusive methods such as X-ray, gamma-ray and ultrasonic techniques have been developed which allow a full determination of the distribution of the dispersed material [15]. In our earlier research, a conceptual model [16] was proposed which described the stability of cement paste as the changing process of different zones. Several parallel test methods including HYdroStatic Pressure Test (HYSPT), in situ volume fraction measurements and total bleeding test were introduced for matrix stability study [16, 17]. HYSPT relies on the fact that the mainly restricted Stokesian settlement of particles causes changes in solid fraction and fresh density at a given height and time. During particle sedimentation, the hydrostatic pressure can be calculated in accordance with Eq. (1) [16], assuming a homogeneous zone below a sharp bleeding front:

$$
p=\rho_{l} \cdot g \cdot h_{b}+\rho_{m} \cdot g \cdot\left(h-h_{b}\right)=\rho_{l} \cdot g \cdot h_{b}+\left[\rho_{s} \Phi+\rho_{l}(1-\Phi)\right] \cdot g \cdot\left(h-h_{b}\right)
$$

Where $p(\mathrm{~Pa})$ is the pressure, $h$ and $h_{b}(\mathrm{~m})$ are the middle depth and bleeding height respectively, $\rho_{l} \rho_{s}$ and $\rho_{m}$ are the density of the liquid, solid and mixture respectively. The mixture between the bleeding front and the middle test level is assumed to be homogeneous with solid fraction $\Phi$ [16]. It can be calculated by inserting the pressure and bleeding measurement results into Eq. (1), see Eq. (2).

$$
\Phi=\left(p-\rho_{l} g h\right) /\left[\left(\rho_{s}-\rho_{l}\right) \cdot g \cdot\left(h-h_{b}\right)\right]
$$

The scope of this research was to investigate the effect on the sedimentation process of adding and replacing two different fillers in pastes of w/c $=0.5$ and 0.7 when combined with various water reducers. Bleeding, hydrostatic pressure and zeta-potential were measured to study how the interactions between particles affect the effective sinking velocity.

\section{MATERIALS AND EXPERIMENTS}

\section{$2.1 \quad$ Materials}

An ordinary Portland cement, CEM I 42.5 in accordance with EN 197-1, was used for the experiments. Two different fillers, crushed Årdal and limestone, were applied both as partial replacement for, and as addition to the cement. The limestone filler consists of almost 85\% calcite in addition to clay minerals. The Årdal filler originates from glaciouflovial aggregate and mostly consists of granite and gneiss. The main minerals are feldspars (plagioclase and potassium feldspar) (approx. 65\%), quartz (approx. 18\%) and mica (approx. 10\%), in addition to some chlorite and amphibolite. The oxide compositions of these two fillers and the cement are shown in Table 1. The particle size distributions of the cement and the fillers determined by a Mastersizer laser granulometer are shown in Figure 1. The limestone powder is much finer than the Årdal filler, but coarser than the cement particles. This is also reflected in their respective Blaine values. Two different types of water reducer, polycarboxylate (PCE) and lignosulfonate $(L S)$ were applied. Table 2 gives the main characteristics of the water reducers. The dosages for all the samples are the solid of the water reducer by the mass of powder including cement and filler ( $s b w p$, solid by the weight of the powder), see Table 3. 
Table 1 - The oxide compositions of the powder materials detected by $X$-ray fluorescence (XRF).

\begin{tabular}{|l|c|c|c|}
\hline Compositions & STD-CEM I & Limestone & Årdal \\
\hline $\mathrm{SiO}_{2}$ & $20.98 \%$ & $16.03 \%$ & $63.56 \%$ \\
\hline $\mathrm{Al}_{2} \mathrm{O}_{3}$ & $5.13 \%$ & $3.29 \%$ & $15.21 \%$ \\
\hline $\mathrm{Fe}_{2} \mathrm{O}_{3}$ & $5.13 \%$ & $2.39 \%$ & $6.05 \%$ \\
\hline $\mathrm{CaO}$ & $60.61 \%$ & $76.40 \%$ & $2.66 \%$ \\
\hline $\mathrm{MgO}$ & $2.39 \%$ & $2.09 \%$ & $2.21 \%$ \\
\hline $\mathrm{P}_{2} \mathrm{O}_{5}$ & $0.14 \%$ & - & $0.28 \%$ \\
\hline $\mathrm{K}_{2} \mathrm{O}$ & $0.86 \%$ & $0.76 \%$ & $4.14 \%$ \\
\hline $\mathrm{Na}_{2} \mathrm{O}$ & $0.43 \%$ & $0.34 \%$ & $3.34 \%$ \\
\hline $\mathrm{SO}_{3}$ & $3.01 \%$ & $0.50 \%$ & - \\
\hline $\mathrm{Blaine}_{3}\left[\mathrm{~m}^{2} / \mathrm{kg}\right]$ & 382 & 361 & 85.6 \\
\hline $\mathrm{D}_{50}[\mu \mathrm{m}]$ & 13.3 & 17.9 & 52.8 \\
\hline $\mathrm{Specific}_{2}$ weight $\left[\mathrm{g} / \mathrm{cm}^{3}\right]$ & 3.15 & 2.75 & 2.68 \\
\hline
\end{tabular}

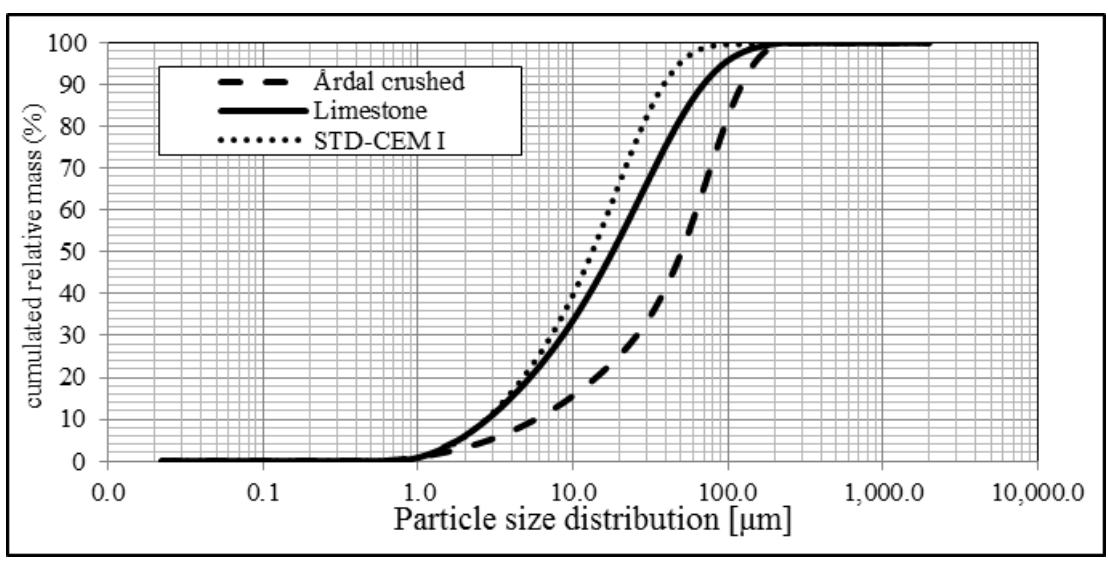

Figure 1 - The particle size distributions of the cement and filler powders.

Table 2 - The main characteristics of the water reducers.

\begin{tabular}{|l|r|r|}
\hline Properties & \multicolumn{1}{|c|}{ PCE } & \multicolumn{1}{c|}{$L S$} \\
\hline Viscosity $^{*}(\mathrm{mPa} \cdot \mathrm{s})$ & Low, $<30$ & Low, $<30$ \\
\hline Solid content & $30.0 \pm 1.5$ & $39.0 \pm 2.0$ \\
\hline Specific gravity $\left(\mathrm{g} / \mathrm{cm}^{3}\right)$ & $1.09 \pm 0.02$ & $1.20 \pm 0.03$ \\
\hline $\mathrm{pH}$-value & $6.5 \pm 1$ & $8.0 \pm 1$ \\
\hline Chloride content (\%) & $<0.01$ & $\leq 0.1$ \\
\hline Alkali $\left(\mathrm{Na}_{2} \mathrm{O}\right.$ equivalent) (\%) & $<2.5$ & $\leq 6$ \\
\hline *Viscosity is measured by Brookfield Viscometer, DV-1, LV1, $100 \mathrm{rpm}, 20 \pm 2{ }^{\circ} \mathrm{C}$ \\
\hline
\end{tabular}

Three liters of cement pastes or matrixes with filler addition were prepared for both HYSPT and bleeding measurements. The mixtures were made using a Hobart mixer with the following mixing procedure: the dry powder was mixed at low speed (591 rpm) for $1 \mathrm{~min}$; water and SP were added simultaneously and mixed for $2 \mathrm{~min}$ at lower speed; then continued mixing at 
middle speed (1485 rpm) for $1 \mathrm{~min}$; waited for $5 \mathrm{~min}$ while using spatula and hand to check the dispersing status and reduced the agglomerates; finally mixed at middle speed for $1 \mathrm{~min}$. All experiments started about 10 min after water addition.

\section{$2.2 \quad$ Experiments}

\section{a) HYSPT}

HYSPT was used to investigate the sedimentation of the matrices. Figures 2 and 3 show HYSPT system setup with two stationary positions for $p(t, h)$ at the depths $h_{b}=200 \mathrm{~mm}$ and $h_{m}=110$ $\mathrm{mm}$. The pressure sensors (Endress\&Hauser) have an accuracy of $0.75 \mathrm{~Pa}$. They automatically correct for atmospheric pressure variations giving only the hydrostatic pressure contribution. More details about the measurement method can be found in our previous papers [16, 17].

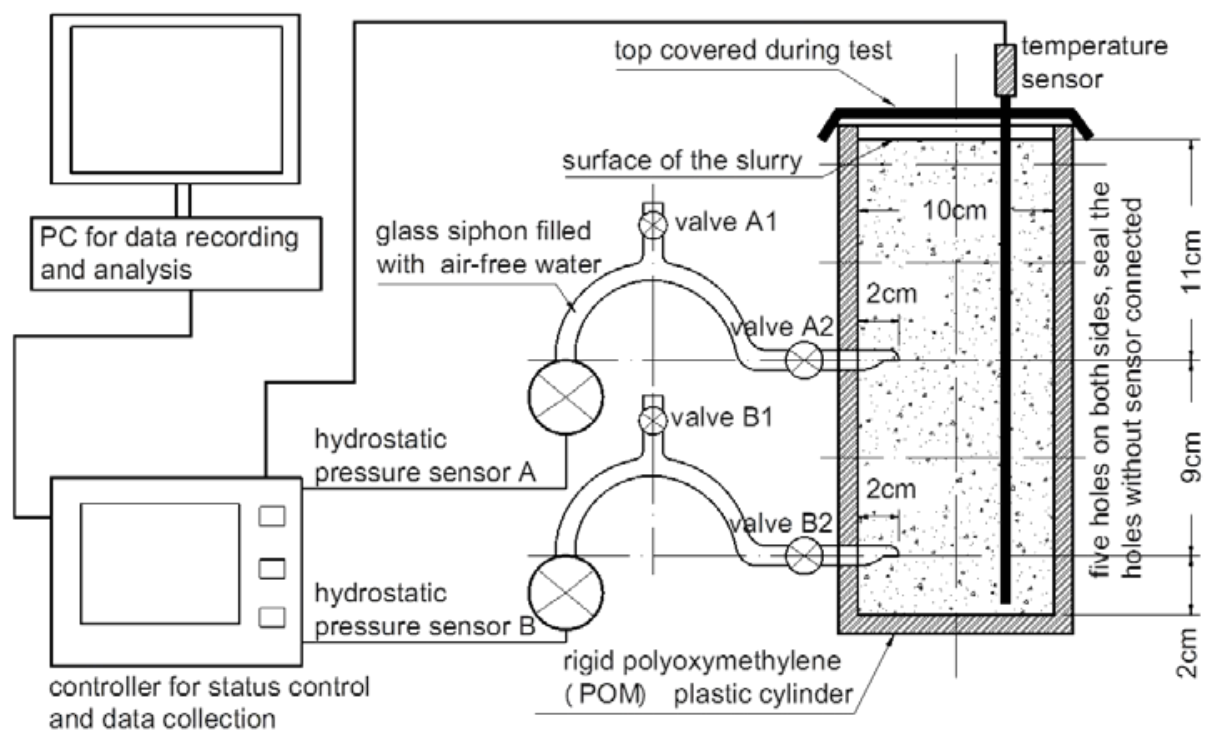

Figure 2 - HYSPT diagram for sedimentation measurements [16].

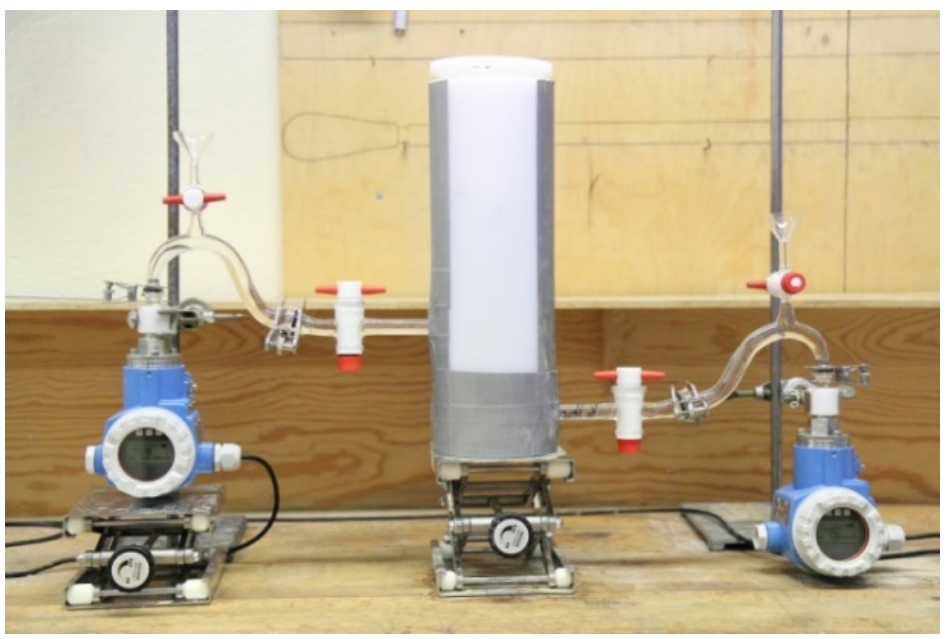

Figure 3 - HYSPT measuring system setup [16]. 
The recipes for the HYSPT measurements are shown in Table 3. The proportioning of cementitious materials for industry use is normally done on a mass basis. Therefore, the proportioning in this research was also done by keeping w/c constant at 0.50 and 0.70 and replacing cement with filler on a mass basis. However, from Eq. (1) and R-Z Equation it can be seen that the volume based fraction is an important factor for investigating the particle sedimentation and bleeding. Two different fillers, crushed Årdal and limestone, were applied either as additions to reduce w/p or as replacement for the cements (approximately constant $w / p$ whereas $w / c$ increased). The small variation in $w / p$ ratio at the same volume-based filler addition was due to the density difference between Årdal and limestone. So the main variables at similar or constant solid fraction were the type of filler and/or admixture.

Table 3 - The recipes of all the cement matrices for HYSPT and visual bleeding measurements.

\begin{tabular}{|c|c|c|c|c|c|c|c|c|c|}
\hline \multirow{2}{*}{ Matrix code } & \multicolumn{2}{|c|}{$\begin{array}{l}\text { Mass based } \\
\text { fractions }\end{array}$} & \multirow{2}{*}{$\begin{array}{l}\text { Filler } \\
\text { types }\end{array}$} & \multirow{2}{*}{\begin{tabular}{|c|} 
Add.f./c \\
mass \\
based \\
\end{tabular}} & \multirow{2}{*}{$\begin{array}{c}\text { Av. powder } \\
\text { density } \\
\left(\rho, \mathrm{kg} / \mathrm{m}^{3}\right) \\
\end{array}$} & \multicolumn{2}{|c|}{$\begin{array}{c}\text { Volume based } \\
\text { fraction }\end{array}$} & \multicolumn{2}{|c|}{ Water reducer } \\
\hline & $\mathrm{w} / \mathrm{c}$ & $\mathrm{w} / \mathrm{p}$ & & & & $\Phi$ & $\mathrm{Vf} / \mathrm{Vp}$ & type* & dosage* \\
\hline wc0.5wp0.5 & 0.50 & 0.500 & & & 3150 & 0.388 & & & \\
\hline wc 0.7 wp 0.7 & 0.70 & 0.700 & & & 3150 & 0.312 & & & \\
\hline wc0.5wp0.5PCE0.18 & 0.50 & 0.500 & & & 3150 & 0.388 & & PCE & $0.18 \%$ \\
\hline ård-wc0.7wp0.585PCE0.18 & 0.70 & 0.585 & Årdal & $20 \%$ & 3063 & 0.358 & $18.5 \%$ & PCE & $0.18 \%$ \\
\hline ård-wc0.7wp0.5 & 0.70 & 0.503 & Årdal & $40 \%$ & 3003 & 0.397 & $31.2 \%$ & & \\
\hline ård-wc0.7wp0.5PCE0.18 & 0.70 & 0.503 & Årdal & $40 \%$ & 3003 & 0.397 & $31.2 \%$ & PCE & $0.18 \%$ \\
\hline ård-wc0.7wp0.5LS0.18 & 0.70 & 0.503 & Årdal & $40 \%$ & 3003 & 0.397 & $31.2 \%$ & LS & $0.18 \%$ \\
\hline lim-wc0.7wp0.5 & 0.70 & 0.500 & limestone & $40 \%$ & 3025 & 0.397 & $31.2 \%$ & & \\
\hline lim-wc0.7wp0.5PCE0.18 & 0.70 & 0.500 & limestone & $40 \%$ & 3025 & 0.397 & $31.2 \%$ & PCE & $0.18 \%$ \\
\hline lim-wc0.7wp0.5LS0.18 & 0.70 & 0.500 & limestone & $40 \%$ & 3025 & 0.397 & $31.2 \%$ & LS & $0.18 \%$ \\
\hline *Notes: & & . & & & 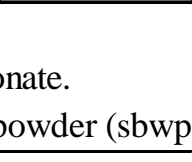 & & & & \\
\hline
\end{tabular}

\section{b) Bleeding}

The bleeding of all HYSPT samples was measured visually, as shown in Figure 4. The experiments were done by filling samples from the same batch of mixtures as for HYSPT into a graduated glass cylinder with $65 \mathrm{~mm}$ diameter and measuring the depth of the visible bleeding water after $4 \mathrm{~h}$. The filling height of samples for the bleeding measurements was the same as for the HYSPT. The cylinder was kept covered with sealing tape during the test to avoid evaporation.

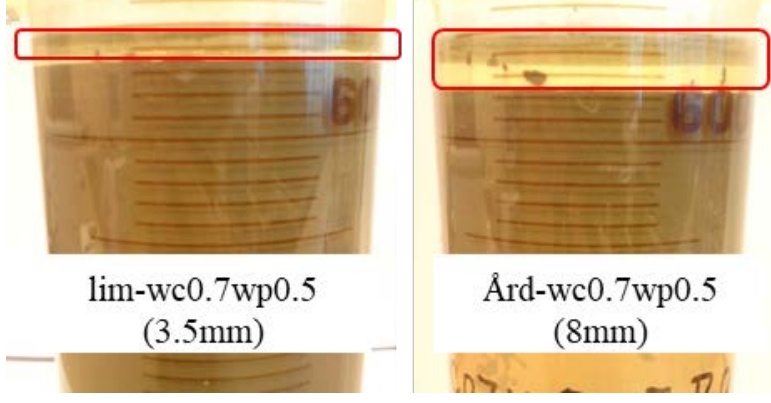

Figure 4 - The visual bleeding of the matrices with fillers (left with limestone; right with Ardal), see the water layer shown in red box. 


\section{c) Zeta-potential}

The zeta-potential of the above two different filler particles, limestone and Årdal, were quantified by the electroacoustic method [18], applying a model DT1200 Electroacoustic Spectrometer from Dispersion Technology Inc. (Bedford Hills, NY/USA). The principle of this method was based on ultrasound propagation through the medium, resulting in an electroacoustic phenomenon called colloid vibration current, from which the zeta-potential is then calculated. The electroacoustic technique characterized the dynamic mobility of particles in suspension. Usually, this technique was used to measure stable homogeneous suspensions with spherical particles (1 nm to $10 \mu \mathrm{m})$. An electric field with frequency around $106 \mathrm{~Hz}$ was applied to the samples, causing charged particles to oscillate, and to produce a sound wave at the same frequency. The oscillation (dynamic mobility) of the particles was described by its magnitude and phase angle (how far the particle motion lags behind the field applied). The sound wave was detected and analyzed to determine the motion of the particles. The main advantage of this technique over "traditional" electrophoretic apparatus is its ability to measure zeta-potential in slurries of greater than $10 \% \mathrm{wt}$. solids concentration, up to $60 \% \mathrm{wt}$. ( $=33 \% \mathrm{vol}$. for a relative solid powder density of $3000 \mathrm{~kg} / \mathrm{m}^{3}$ suspended in water) depending on the sample.

Table 4 - The mixtures for zeta-potential measurements.

\begin{tabular}{|c|c|c|c|c|c|c|c|c|c|c|c|}
\hline \multirow[b]{2}{*}{ No. } & \multirow[b]{2}{*}{ group } & \multirow[b]{2}{*}{ Recipes ${ }^{* 1}$} & \multirow{2}{*}{\begin{tabular}{c|} 
Cement \\
{$[\mathrm{g}]$}
\end{tabular}} & \multicolumn{2}{|c|}{ Filler } & \multicolumn{3}{|c|}{ Water reducer ${ }^{* 3}$} & \multirow[b]{2}{*}{$\mathrm{w} / \mathrm{c}$} & \multirow{2}{*}{$\begin{array}{c}\text { Apparent } \\
\mathrm{w} / \mathrm{p}^{* 4}\end{array}$} & \multirow[b]{2}{*}{ Medium } \\
\hline & & & & type & $\begin{array}{c}\text { amount } \\
{[\mathrm{g}]}\end{array}$ & type & $\begin{array}{c}\text { amount } \\
{[\mathrm{g}]}\end{array}$ & $\begin{array}{c}\text { amount } \\
\text { [sbwp, \%] }\end{array}$ & & & \\
\hline 1 & \multirow{3}{*}{ group I } & Cem+water & 50 & - & - & - & - & - & 0.5 & 0.5 & water \\
\hline 2 & & Cem+LS & 50 & - & - & LS & 0.3 & 0.24 & 0.5 & 0.5 & water \\
\hline 3 & & Cem+PCE & 50 & - & - & PCE & 0.3 & 0.18 & 0.5 & 0.5 & water \\
\hline 4 & \multirow{8}{*}{$\begin{array}{c}\text { group } \\
\text { II }\end{array}$} & Lim+water & - & Lim & 50 & - & - & - & - & 0.57 & water \\
\hline 5 & & $\mathrm{Lim}+(\mathrm{Cem}-\mathrm{PS})$ & - & Lim & 16.8 & - & - & - & 0.67 & 0.5 & Cem-PS \\
\hline 6 & & Lim+LS (Cem-PS) & - & Lim & 50 & LS & 0.3 & 0.24 & $0.5^{* 2}$ & 0.57 & Cem-PS \\
\hline 7 & & Lim+PCE (Cem-PS) & - & Lim & 50 & PCE & 0.3 & 0.18 & $0.5^{* 2}$ & 0.57 & Cem-PS \\
\hline 8 & & Ård+water & - & Ård & 50 & - & - & - & - & 0.57 & water \\
\hline 9 & & Ård+(Cem-PS) & - & Ård & 16.8 & - & - & - & 0.67 & 0.5 & Cem-PS \\
\hline 10 & & Ård+LS (Cem-PS) & - & Ård & 50 & LS & 0.3 & 0.24 & $0.5^{* 2}$ & 0.57 & Cem-PS \\
\hline 11 & & Ård+PCE (Cem-PS) & - & Ård & 50 & PCE & 0.3 & 0.18 & $0.5^{* 2}$ & 0.57 & Cem-PS \\
\hline 12 & \multirow{4}{*}{$\begin{array}{c}\text { group } \\
\text { III }\end{array}$} & Lim+Cem+LS & 33.2 & Lim & 16.8 & - & - & - & 0.67 & 0.5 & water \\
\hline 13 & & Lim+Cem+PCE & 33.2 & $\operatorname{Lim}$ & 16.8 & 0.3 & 0.3 & 0.18 & 0.67 & 0.5 & water \\
\hline 14 & & Ård+Cem+LS & 33.2 & Ård & 16.8 & - & - & - & 0.67 & 0.5 & water \\
\hline 15 & & Ård+Cem+PCE & 33.2 & Ård & 16.8 & 0.3 & 0.3 & 0.18 & 0.67 & 0.5 & water \\
\hline \multirow{4}{*}{ Notes } & \multicolumn{11}{|c|}{ *1: Cem-cement, Lim-limstone, Ård-Årdal; } \\
\hline & \multicolumn{11}{|c|}{ *2: the w/c with $* 2$ means the w/c ratio only used for the pore solution medium. } \\
\hline & \multicolumn{11}{|c|}{ *3: the dosage of all the water reducer refer to solid by the weight of the powder. } \\
\hline & \multicolumn{11}{|c|}{ *4: Because the cement was used to prepare the pore solution while the filler and water reducer were added } \\
\hline
\end{tabular}

The zeta-potential for the samples containing only cement was measured on samples prepared with deionised water. For the samples containing filler without water reducer, cement pore solution was used. The cement pore solution was obtained by filtering (1) standard cement slurries, (2) filler slurries or (3) cement + filler slurries using a Büchner funnel with cellulose filter paper and connected to a water pump setup. The w/c of the suspensions was kept at 0.5 when preparing the cement pore solution. For each batch of pore solution, $400 \mathrm{~g}$ of dry powder was employed to minimize any systematic error which could arise as a result of differences in suction force on samples based on differences in their weight. The yield of pore solution per batch of suction filtration was less than $100 \mathrm{~g}$. The pore solutions were used almost immediately 
and stored under vacuum to reduce the risk of carbonation. Prior to the zeta-potential measurements, all dry powders (cement and fillers when applicable) were weighed out in a 50 $\mathrm{ml}$ PE tube. The total amount of dry powder for each preparation was kept at a constant amount of $50 \mathrm{~g}$. Water or pore solution at the desired water to filler ratio (w/f) was added to the dry powder and the sample was mixed in a vortex for $15 \mathrm{~s}$. The homogenized mixture was subsequently poured into a $15 \mathrm{~mL}$ beaker, where the zeta-potential values were measured within $3 \mathrm{~min}$ after wetting. Where required, the water reducers were mixed into the solution before adding it to the dry powder. The amount of materials and the type of medium (water or pore solution) employed for each measurement can be found in Table 4. High concentrations of filler were not used in this study to avoid excessive sedimentation of particles that could affect the zeta-potential measurements.

\section{RESULTS AND DISCUSSION}

\subsection{Bleeding}

Table 5 enables comparison of the bleeding depths of the suspensions shown in Table 3 at $4 \mathrm{~h}$ after mixing. The paste with higher w/c ratio (i.e. low solid fraction) without water reducer showed much higher bleeding. No clear bleeding could be observed for the paste or matrices with $0.18 \%$ PCE. The top of these matrices contain a diluted muddy layer (see Figure 5 ), instead of the transparent bleeding water that could be seen with the same dosage of LS (Figure 6). This could be due to the different dispersing efficiency between LS and PCE. When the particles are better dispersed in matrices with PCE, the particles sink slower or even remain suspended.

Table 5 - The visible bleeding depth of the matrices for HYSPT at $4 \mathrm{~h}$ after mixing.

\begin{tabular}{|c|l|c|}
\hline No. & \multicolumn{1}{|c|}{ matrix code } & visible bleeding [mm] \\
\hline 1 & wc0.5wp0.5 & 2.5 \\
\hline 2 & wc0.7wp0.7 & 28.0 \\
\hline 3 & wc0.5wp0.5PCE0.18 & $/$ \\
\hline 4 & ård-wc0.7wp0.585PCE0.18 & $/$ \\
\hline 5 & ård-wc0.7wp0.5 & 8.0 \\
\hline 6 & ård-wc0.7wp0.5PCE0.18 & $/$ \\
\hline 7 & ård-wc0.7wp0.5L S0.18 & 12.0 \\
\hline 8 & lim-wc0.7wp0.5 & 3.5 \\
\hline 9 & lim-wc0.7wp0.5PCE0.18 & $/$ \\
\hline 10 & lim-wc0.7wp0.5LS0.18 & 6.5 \\
\hline
\end{tabular}

The matrix with Årdal filler, displays higher bleeding depth than the matrix with limestone, both with and without admixture, compare No. 5 with No. 8 and No. 7 with No. 10 (all with the same initial solid fraction), see also Figure 6 . The higher bleeding depth is to be expected because the coarser particle size and lower specific surface area of Årdal filler induces a higher particle sinking velocity and therefore more water flowing upwards. The change of bleeding with water reducer is in line with earlier observations [17] and is presumably due to the smaller effective particle size in a de-flocculated suspension. 


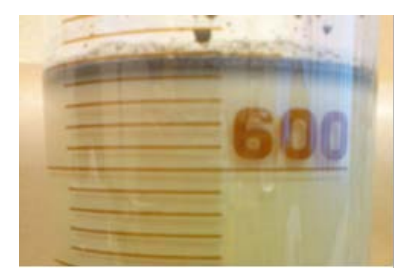

Ård-wc0.7wp0.5PCE0.18 (No clear bleeding front)

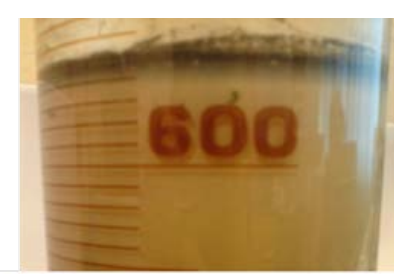

lim-wc0.7wp0.5PCE0.18 (No clear bleeding front)

Figure 5 - No clear bleeding was observed for the matrices with filler and 0.18\% PCE.

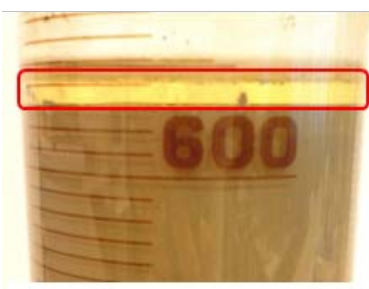

lim-wc0.7wp0.5LS0.18 $(6.5 \mathrm{~mm})$

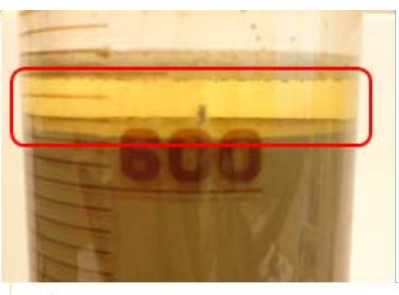

Ård-wc0.7wp0.5LS0.18 (12mm)

Fiure. 6 - Visible bleeding was observed for the matrices with filler and 0.18\% LS.

\subsection{Sedimentation of matrices}

a) Reference pastes with and without water reducer

Figure 7 shows the pressure changes at bottom (left plot) and middle (right plot) for pastes of varying $w / p$ ratio without water reducer. The differences between the starting points for the two materials in each plot show the different initial pressure due to different densities and heights of the suspensions in the container (shown in Figure 2). The paste with higher $\Phi(w / p=0.5)$ shows a slightly larger $d p / d t$ and thus a higher sedimentation rate. This does not coincide with the $R-Z$ $E q$. [8], which predicts that a higher initial solid fraction $\Phi_{0}$ induces lower particle sinking velocity. The most probable reason is that more flocculation occurred in the pastes with higher $\Phi_{0}$, which induces larger "effective" particle sizes and higher particle sinking velocity. At around $2 \mathrm{~h}$, both pastes reach the plateau with a clear inflection point. At the plateau the solid particles above the sensor tip have settled and the pressure detected is the liquid pressure. Fig. 7 shows that both pressure curves reach the plateau pressures close to the pure water pressures of $1100 \mathrm{~Pa}$ in the middle and $2000 \mathrm{~Pa}$ at the bottom. This confirms that the pressure detected is governed by particle sedimentation.

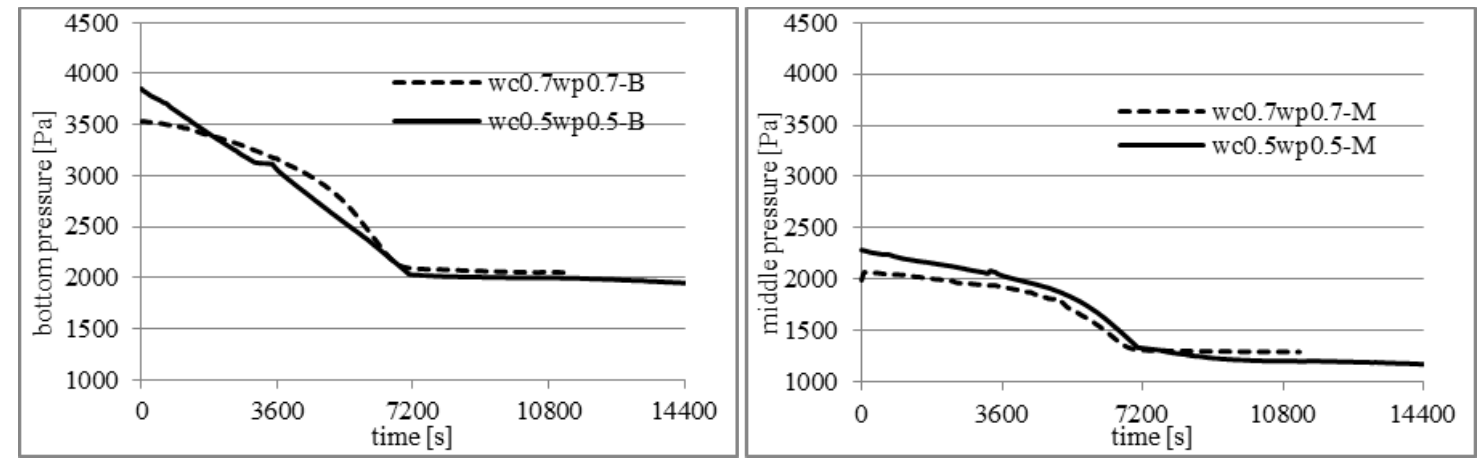

Fiure. 7 - Pressure curves of the pastes with different w/c ratio without water reducer. 
Figure 8 shows the pressure results of the pastes with PCE water reducer. In the first $0.5 \mathrm{~h}$, the matrix with lower $\Phi_{0}$ (wc0.7wp0.7PCE0.18) shows a larger $\mathrm{dp} / \mathrm{dt}$ and a higher sedimentation rate. This coincides with the $R-Z$ Eq. However, the matrix with higher $\Phi_{0}$ (wc0.5wp0.5PCE0.18) presents a higher sedimentation rate after this period and thus does not obey the $R-Z E q$. any longer. This alteration indicates two possible phenomena: either $0.18 \%$ PCE SP is not enough to disperse all the particles for the paste with $w / p=0.5$; or particle flocculation may be delayed by water reducer until after a period of measurement.
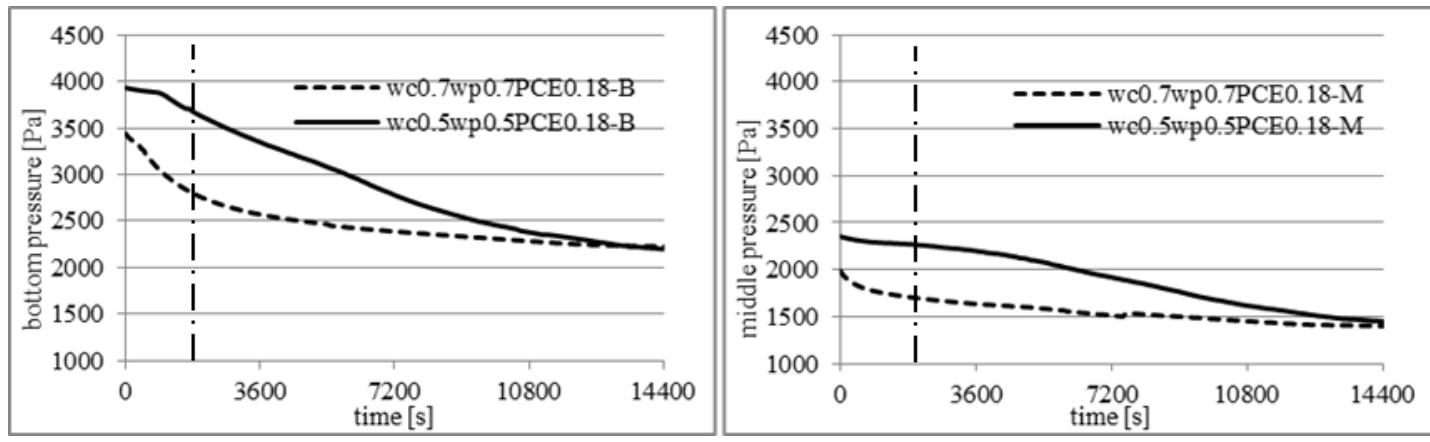

Figure 8 - Pressure curves of the pastes with $w / c=0.5$ and 0.7 and $0.18 \%$ PCE.

\section{b) Filler replacement/addition effect for matrices without water reducer}

The matrices with constant $w / p=0.5$ without water reducer were investigated to check the filler replacement effect, as shown in Figure 9. Note that the matrices with fillers have a bit higher $\Phi_{0}$ (0.397) than that of the paste with $\Phi_{0}=0.388$ due to differences in powder density. The $40 \%$ limestone replacement by mass leads to higher sedimentation rate and takes less time to reach the plateau than the reference cement paste $w c 0.5 w p 0.5$, while the coarser Årdal replacement induces the opposite effect. This indicates that the finer limestone induced higher sedimentation rates, which does not coincide with Stokes law [15]. It is believed that this was caused by more flocculation of the matrix with limestone, see the separate zeta-potential investigation below. Compared with $w c 0.7 w p 0.7$, the additions of the different fillers result in quite different effects. Figure 9 shows that the average $d p / d t$ is slightly higher for the limestone matrix than for the pure paste with $w / c=0.7$. However, comparing $d p / d t$ of $w c 0.7 w p 0.7$ with $a r d-w c 0.7 w p 0.5$, the Årdal matrix shows slightly lower $d p / d t$ and takes a longer time to reach the plateau. This indicates that the Årdal modified matrix had the slowest sedimentation rate.
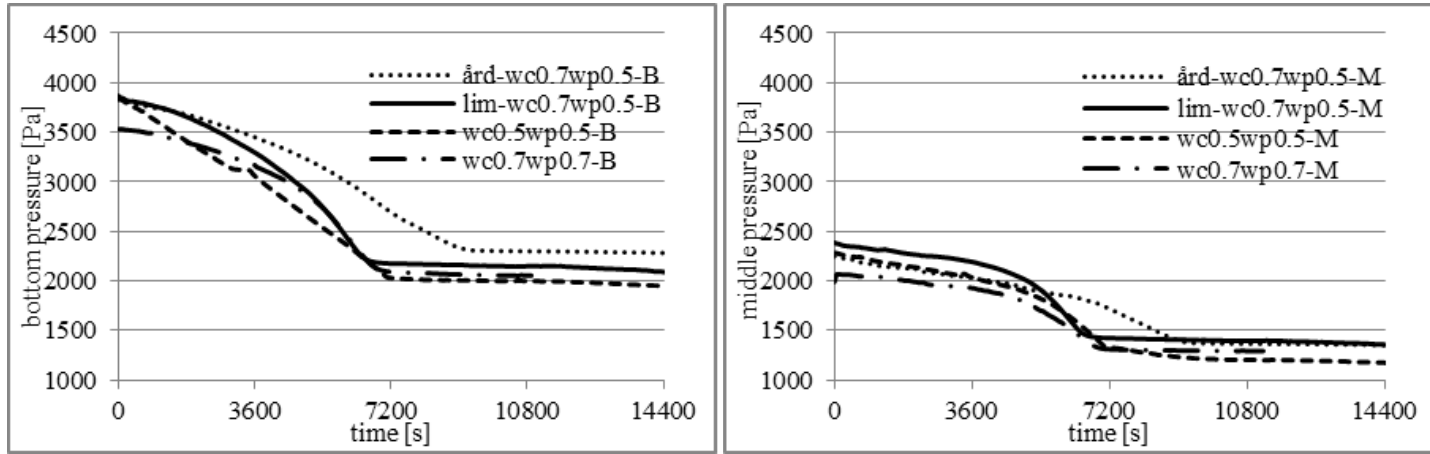

Figure 9 - Pressure curves of the matrices with $w / p=0.7$ or 0.5 by adding various fillers, no water reducer. 


\section{c) Filler effect for matrices with $S P$}

\section{- Filler replacement (constant w/p)}

It has previously been observed [17] that water reducer helps stabilizing the cement paste by dispersing the particles, which reduces the effective particle size and delays the early hydration of cement. Figure 10 shows the sedimentation behavior of matrices with limestone or Årdal filler and with $0.18 \%$ solid dosage of PCE water reducer $\left(\Phi_{0}=0.397\right)$ compared with the paste with the same SP $\left(\Phi_{0}=0.388\right)$. Compared to Figure $9, d p / d t$ is lower and the inflection point less sharp in Figure 10 as more small particles settled. Figure 11 shows that with LS water reducer, there is no longer any difference in the sedimentation behavior of the matrices at the same $\Phi_{0}$ as opposed to Figure 9. It seems that the dosage applied is sufficient to disperse the limestone particles so that the different particles behave in a similar way at $\Phi=0.397$. Compared with the matrices without water reducer shown in Figure 9, it can also be seen that the time periods to reach the plateau were all delayed and a less sharp inflection points appeared for all matrices in Figure 10. Again, the dispersing effect of $0.18 \%$ of PCE, i.e. the normal dosage of this water reducer overshadowed the influence of various fillers on the sedimentation process compared to the matrices without water reducer. Similarly, the two matrices with $L S$ (see Figure 11) show identical $d p / d t$. According to Table 5 , the LS addition at $0.18 \%$ seems to increase the bleeding though only the visual bleeding was taken into account. The matrices with coarser particles, either with or without water reducer, caused higher bleeding as expected from K-C Eq. $[10,11]$. This is why fillers with different particle sizes showed an opposite effect on sedimentation and visual bleeding. It is reasonable because visual bleeding tests did not include the soft turbid bleeding and the solid fraction differences at the zones below bleeding, while HYSPT measurements take solid fraction changes of more zones into account at both levels. This phenomenon will be explained further in Figure 15 below.
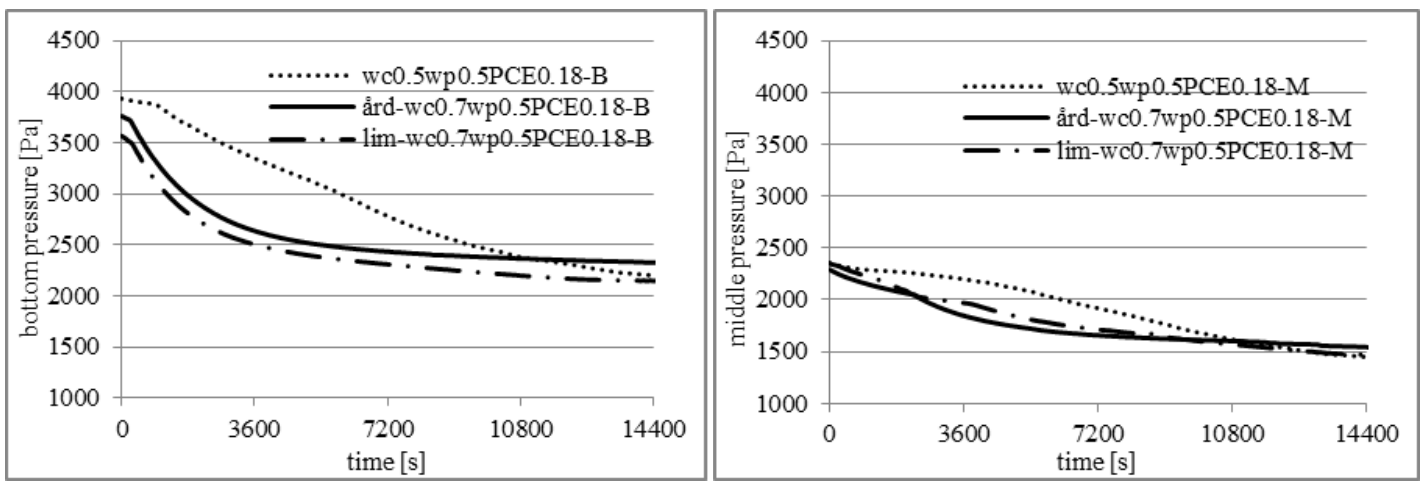

Figure 10 - Pressure curves of paste $\left(\Phi_{0}=0.388\right)$ and two matrices with the same dosage of PCE but with different types of filler $\left(\Phi_{0}=0.397\right)$.
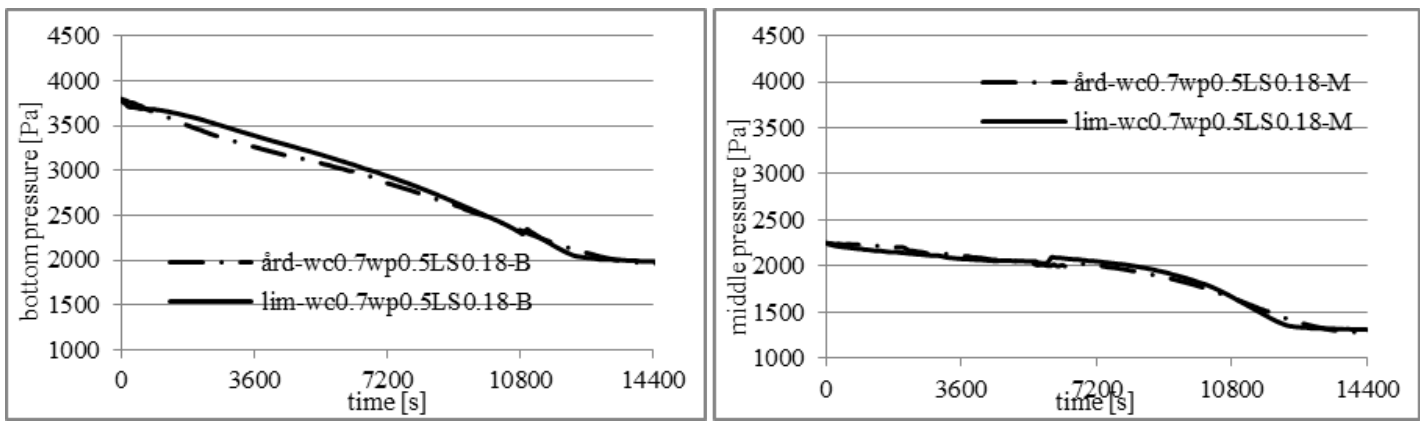

Figure 11 - Pressure curves of two matrices with the same dosage of LS but with different types of filler $\left(\Phi_{0}=0.397\right)$. 


\section{- $\quad$ Filler addition (constant $w / c=0.7)$}

Figure 12 shows the HYSPT results for cement paste $(\Phi=0.312)$ and matrices with two different filler dosages ( $\Phi_{0}=0.358$ and 0.397$)$, all with the same water reducer. There is very little difference in the sedimentation curves. The variation is probably within the expected variation of the test method itself $[16,17]$. This is surprising but indicates that at this $\Phi_{0}$, this dosage of PCE leads to equal states of dispersion. This is a very important factor and could describe the critical dosage of this $S P$ for matrix at this $\Phi_{0}$, with which all the particles get totally dispersed. Visual bleeding tests show that there were no clear bleeding fronts but turbid bleeding zones for both matrices, see Figure 15.

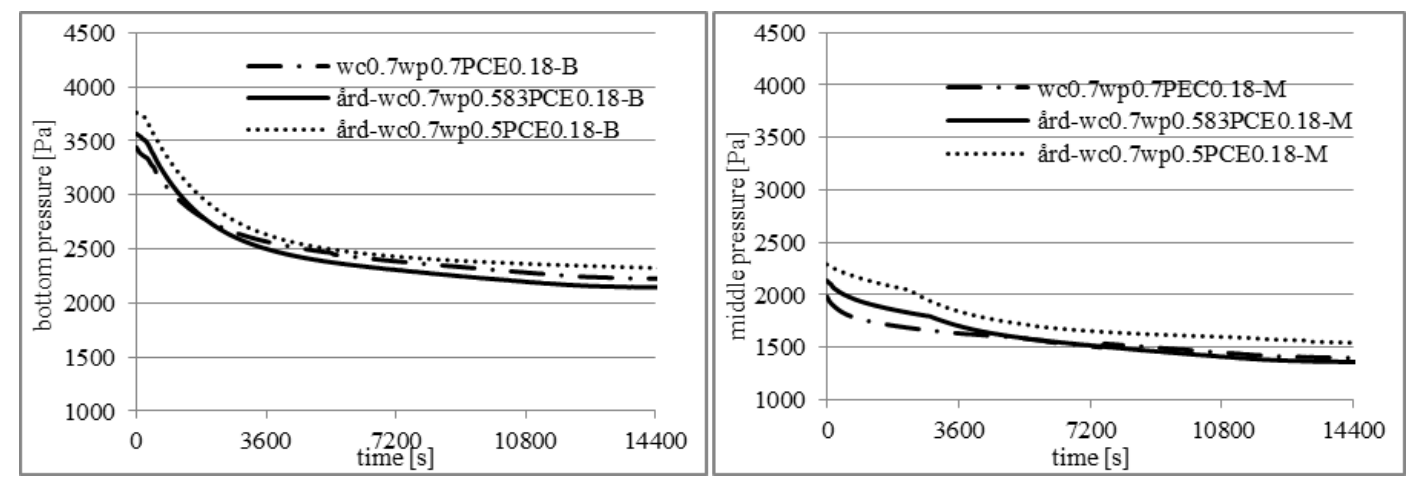

Figure 12 - Pressure curves of paste $\left(\Phi_{0}=0.312\right)$ and two matrices with different percentage of Ardal filler $\left(\Phi_{0}=0.358\right.$ and 0.397$)$ but the same dosage of PCE.

\section{d) The effect of different water reducer}

Figure 13 shows the effects of no water reducer, $L S$ and PCE on the sedimentation of the matrices with limestone filler. Compared with the matrix without water reducer, the matrices with $P C E$ and $L S$ take much longer time to reach a plateau or did not reach it within $4 \mathrm{~h}$. The matrix with $P C E$ displays a very low sedimentation rate during most parts of the sedimentation process. The matrix with LS also takes longer time and has less clear inflection point. This indicates that with the same filler addition, PCE has more effect on slowing down sedimentation due to the better dispersing effect. Previously, these two types of water reducers have been investigated in a plain cement paste with $w / c=0.5\left(\Phi_{0}=0.388\right)$ [17] without noticing any particular effect of changing the type of water reducer.
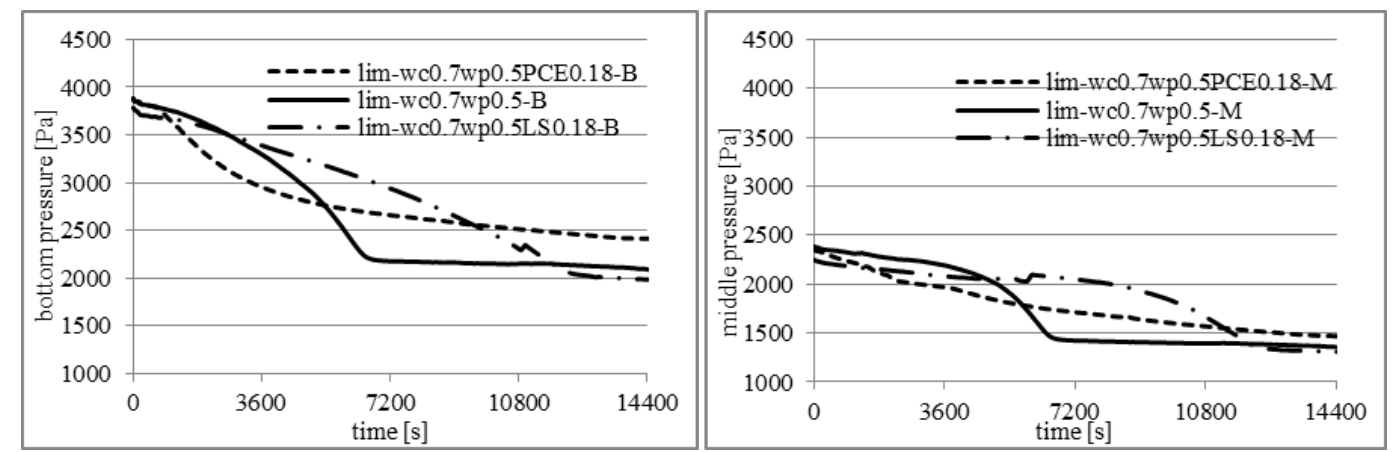

Figure 13 - Pressure curves of matrices with the same limestone addition without and with 2 different water reducers at $0.18 \%$.

A conceptual model for sedimentation and bleeding of cement paste was proposed in the previous paper [16]. It describes the process to be variation of zones including supernatant (bleeding), homogeneous zone, variable concentration and sediment cake, as shown in Figure 14. The bleeding observations accentuates the importance of the volume fraction curve ( $\Phi$ - 
curve), which indicates that in stage 1 the transition between bleeding and paste is not a sharp front shown as the various gray scales. According to the photo with high resolution shown in Figure 15, the phenomena observed during experiments seem to coincide qualitatively with the conceptual model.

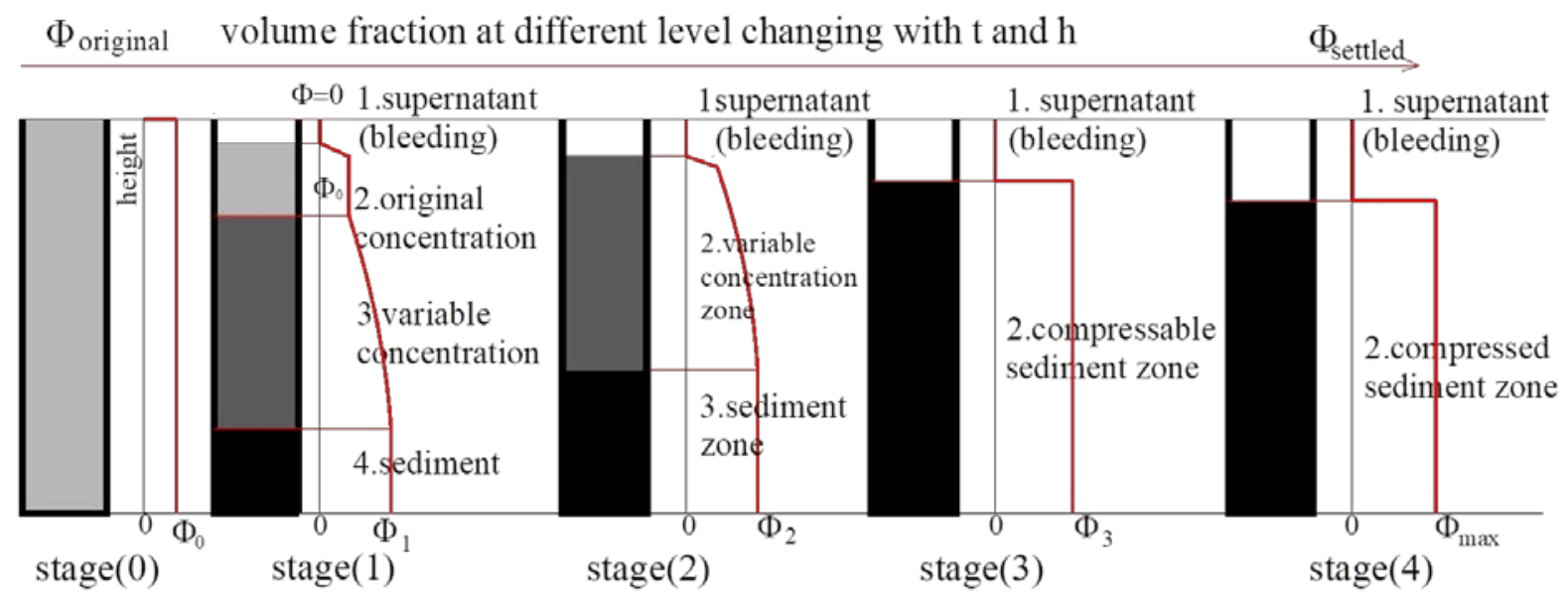

Figure 14 - The conceptual model for stability of cement paste [4, 16].

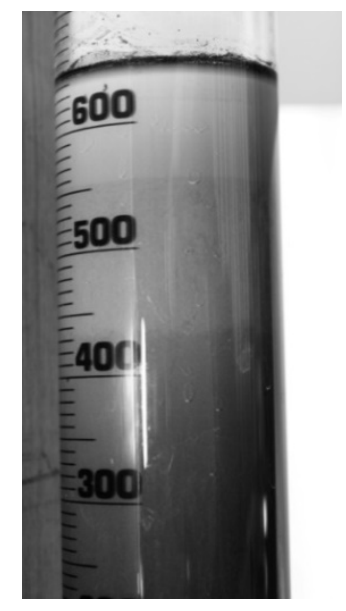

Figure 15 - Zones transformation for lim-wc0.7wp0.5PCE0.18 at $2 \mathrm{~h}$ sedimentation after mixing.

The effect of different water reducers is also compared for the matrices with the Årdal filler, as shown in Figure 16. The features of the curves are similar to those in Figure 13, but the rank of sedimentation differs from the reference. With regard to $d p / d t$ and the time to reach the plateau, Figure 16 shows that the matrix with PCE presents faster sedimentation than that with $L S$. So $L S$ seems to stabilize the Årdal matrix better than PCE water reducer. The reason for the faster sedimentation with PCE could be the different influence of these two water reducers on the inter- the middle level. There could be two reasons for this. Firstly, there are probably particle forces, which will be discussed in section 3.3 below. Moreover, these results only show the sedimentation process within the first $4 \mathrm{~h}$. Figure 16 also shows that the pressure after $4 \mathrm{~h}$ is more than $2000 \mathrm{~Pa}$ at the bottom level and more than $1100 \mathrm{~Pa}$ at tiny particles which settled at a very low velocity or could not sink at all. Secondly, the compression of the sediment cake continues. Our previous research $[4,16,17]$ also found that cement paste with the same PCE showed a gradual pressure reduction after reaching the plateau. The combined effect of $S P$ and filler on compressibility was earlier discussed in terms of paragenesis [17]. 


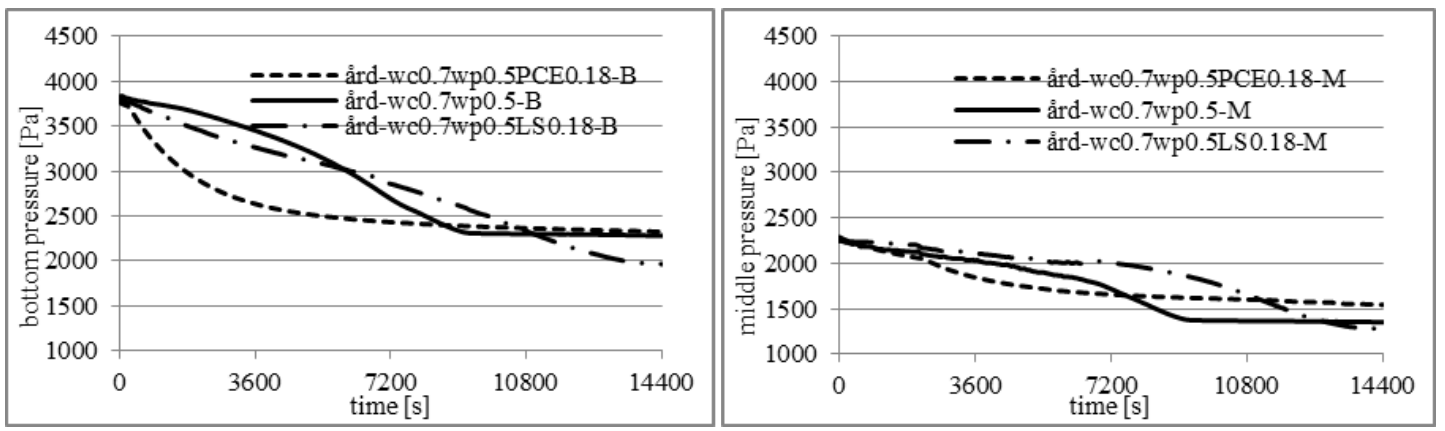

Figure 16 - Pressure curves of the matrices with the same Årdal addition and different water reducers.

\subsection{Combined effect of filler and water reducer on zeta-potential}

a) Effect of water reducer on cement

The zeta-potential results are shown in Figures 17 to 19 in the three different groups as presented in Table 4: (1) cement only, (2) filler only and (3) filler mixed with cement. The zetapotentials of the first group are shown in Figure 17. There are at least two possible explanations for the positive zeta-potential $(+2.2 \mathrm{mV}$ in the pure cement paste with $w / c=0.5)$ : (1) the heterogeneous cement particles is represented by more positively charged clinker phases on the surfaces; (2) the rapid hydration of $\sim 7 \% C_{3} A$ happened in presence of gypsum. Hydration of the aluminate phase leads to the formation of $C A H$ compounds, which carry a positive zeta-potential [19]. Possibly, it could be a combination of with high $\mathrm{Ca}_{2+}$ concentrations in the pore solution which shields the negatively charged surfaces and potentially "recharge" them.

When water reducer was added, the zeta-potential increased slightly to become more positive, which is in line with better dispersion effect by adding water reducer and thus a lowered sedimentation rate as observed by the HYSPT. A possible hypothesis for this observation could be linked to the negative nature of the water reducer and abundant cations such as $\mathrm{Ca}^{2+}$ released in cement pore solutions [20]. The presence of negative water reducer on the cement particles masks their positive surfaces, turns them negative and promoted adsorption of cations onto the polymer to form a double layer. This hypothesis is supported by the fact that the cement pastes with the more anionic $L S$ water reducer show a lower zeta-potential value than that with PCE.

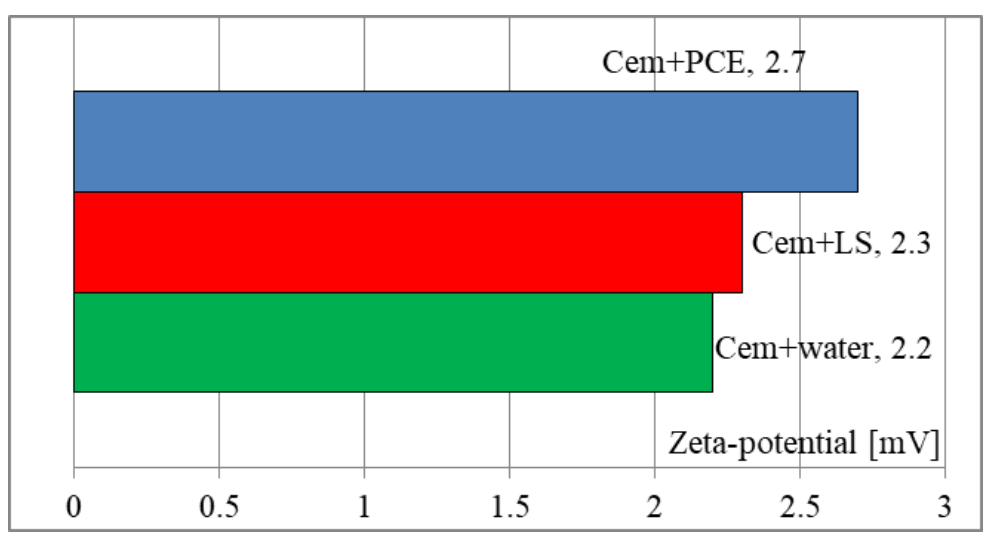

Figure 17 - zeta-potential of group 1 samples - cement paste with and without LS or PCE water reducer, respectively. 


\section{b) Effect of water reducer on fillers}

The fillers were first tested in deionized water to determine the innate surface of the particles. Figure 18 shows that both fillers had a zeta-potential value close to zero: limestone gave a positive value of $+0.8 \mathrm{mV}$, whereas Årdal was negative at $-0.5 \mathrm{mV}$. This renders them less stable than the cement particles, thus the fillers may be more susceptible to flocculation in deionized water, but not in the same environment, i.e. $\mathrm{pH}$ and ionic strength as Cem-PS. When added to cement pore solution, the zeta potentials are rapidly reversed, indicating a quick adsorption of ions, $\mathrm{Ca}^{2+}$ and $\mathrm{SO}_{4}{ }^{2-}$ most likely onto the charged surfaces of the fillers.

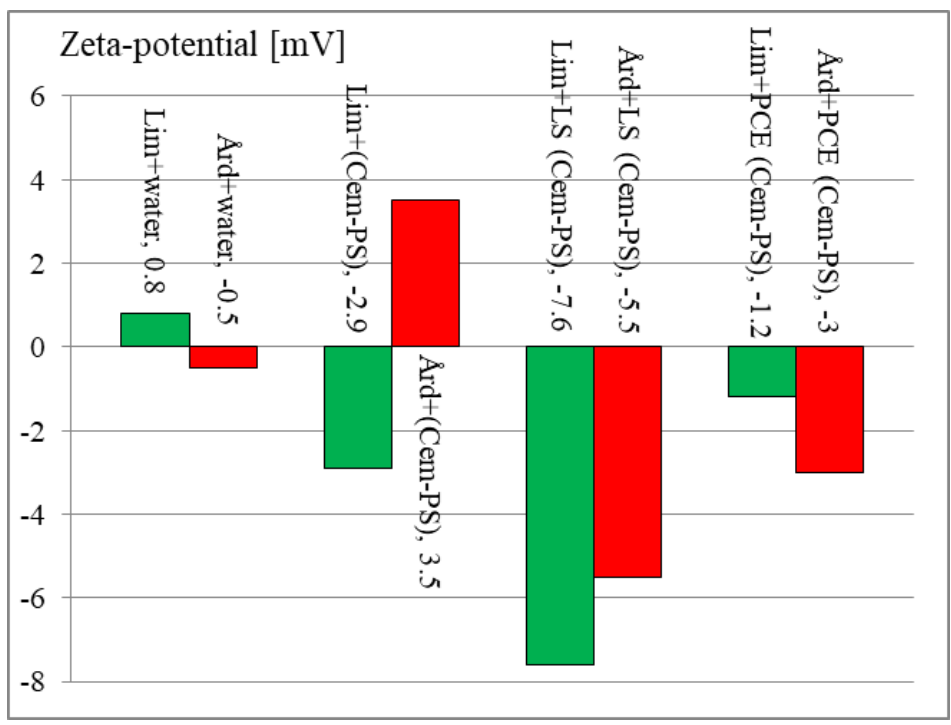

Figure 18 - Zeta-potential of cement paste, limestone and Årdal in water or pore solution with either presence or absence of water reducer.

Due to the rapid uptake of ions, the addition of both fillers does not intensify the flocculation of the matrices lim-wc0.7wp0.5 and ård-wc0.7wp0.5. Figure 18 also shows that both fillers display negative zeta-potentials when adding water reducer, whereby the zeta-potentials of the suspensions with LS are more negative than that with PCE. This can be accounted for the steric bulk or the higher charge density of LS, which enhances the stability of the suspension and thus lower the sedimentation rate as shown in Figure 13. Similar phenomenon can also be seen in the filler modified matrices that will be discussed later.

The two water reducers exert various interesting effects on the fillers. In the presence of LS, limestone suspensions display a more negative zeta-potential value $(-7.6 \mathrm{mV})$ than Årdal suspensions $(-5.5 \mathrm{mV})$. This can be attributed to the initial zeta-potentials of the fillers, whereby an overall less negative zeta-potential in Årdal than by limestone occurs after adsorption. However, $\Delta Z(9 \mathrm{mV})$ between $A r d+(C e m-P S) / A r d+L S(C e m-P S)(\triangle Z=9 \mathrm{mV})$ is greater than that between $\mathrm{Lim}+(\mathrm{Cem}-P S) / \mathrm{Lim}+\mathrm{LS}(\mathrm{Cem}-\mathrm{PS})(\triangle Z=4.7 \mathrm{mV})$. This suggests that a higher amount of $L S$ is adsorbed by Årdal than by limestone, which could lead to LS having a better dispersing effect on Årdal. In general, the higher the zeta-potential value (+/-), the more stable fillers in the polyelectrolyte rich medium would be, due to the better dispersion of the particles. This could be the reason for the lower sedimentation rates of matrices with LS than those of matrices with PCE, as shown in Figure 13 and 16.

c) Cement mixed with filler in presence of water reducers

Figure 19 shows zeta-potential results of suspension group III (see Table 4) for the combination of filler and cement in a water reducer dispersed suspension. Based on their polarity, both the cement and fillers are potential docking sites for the adsorption of the water reducer, which in turn effectively varies the zeta-potential of the suspension. The zeta-potential values of the 
matrices with LS are lower than for pure filler suspensions due to the inherent positive nature of the cement suspension. Such negative zeta-potential also indicates that LS adsorbed onto the filler equally rapidly as on to cement particles, which allows the zeta-potential of the matrices with both types of fillers to be negative. However, the change of filler from limestone to Årdal in the matrices with PCE made the zeta-potential vary from +0.2 to $-1.8 \mathrm{mV}$. This indicates that PCE may preferentially adsorb onto cement particles for the matrices with limestone, while competitive adsorption of PCE between cement and Årdal may happen due to the highly positive surface charge of Årdal filler after $S P$-adsorption (see Figure 18). In group III of Table 4, the suspensions with $L S$ shows similar negative zeta-potential values, which match with the similar sedimentation rate between matrices with $L S$ and various fillers, as shown in Figure 11.

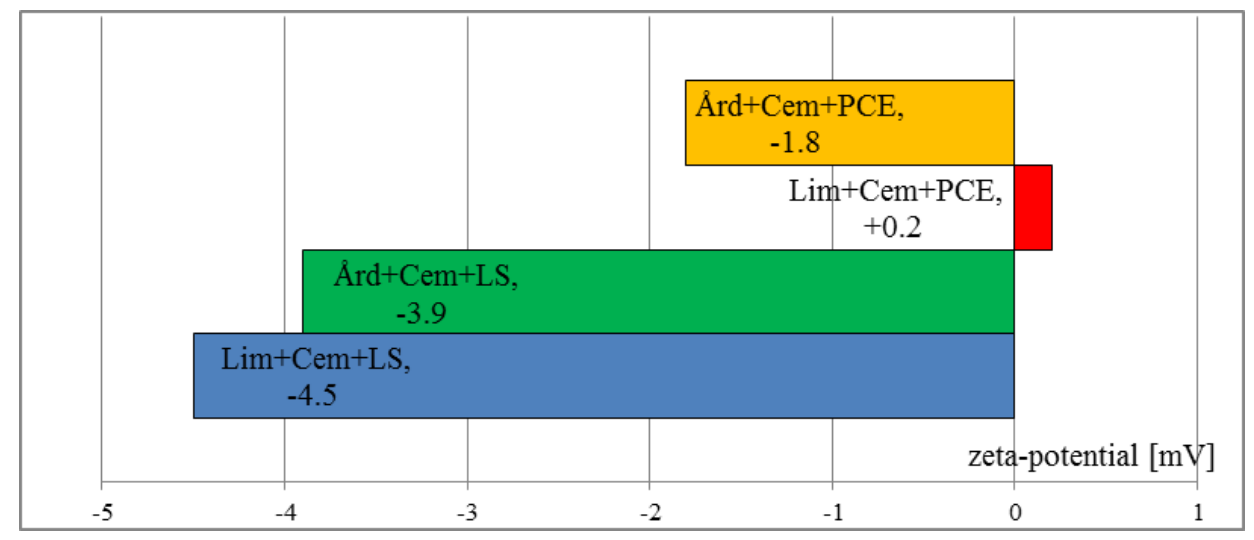

Figure 19 - zeta-potential of the combined filler and cement particles in pore solutions with presence of LS or PCE.

Considering the various zeta-potential values of the matrices with PCE, the addition of Årdal or limestone filler should present very different effects on particle dispersion and therefore the sedimentation process. However, both Figures 10 and 12 show that the addition of various fillers brings about a similar effect on sedimentation when combined with same PCE. This seemingly contradictory effect can be related to the following three facts. Firstly, the steric effect needs to be taken into account for the matrices with PCE-based water reducer. It can greatly alter the dispersion and reduce flocculation in the matrices which appears to have low stability in zetapotential measurements. Moreover, the preferential adsorption of PCE onto cement particles for the matrices with limestone may further increase the overall dispersity of the suspensions. Finally, the w/c ratios of the samples in group III are higher than those for HYSPT, which might partially explain the contradictory effect of the filler on sedimentation and zeta-potential. In general, the matrices with LS displayed a larger absolute value of the zeta-potential than those with PCE. This again indicates lower tendency to particle flocculation in the samples with $L S$ and resulted in lower sedimentation rates, as can be seen in Figure 13 and 16. After the initial few minutes, the results are relatively stable for all samples in the zeta-potential measurements. Small changes are observed in measurements up to $10 \mathrm{~min}$. This indicates two points: firstly the overall variation of the filler surfaces is minimal after the initial change; secondly the adsorption of water reducer on the particle surfaces is rapid and stable as long as no external factor presents to influence this attraction. 


\subsection{Final discussion}

The bleeding results show that the solid fraction and specific surface of the particles could be the main parameters in line with the K-C Eq. [10]: the highest and lowest bleeding (28 $\mathrm{mm}$ and $2.5 \mathrm{~mm}$ as shown in Table 5) are for the pure pastes with large difference in solid fraction and equal particle fineness. However, the two reference pastes show similar sedimentation processes. This indicates that the total bleeding- and sedimentation measurements might not separately demonstrate the same stabilizing effect. This is in line with the findings [21] on fundamental differences between total bleeding- and hydrostatic pressure measurements and how bleeding rates and $d p / d t$ close to the surface relate. It should also be noted that only the transparent bleeding could be observed in the visual bleeding tests. For the cases with PCE, a long compressing process which did not reach a plateau was observed. A turbid bleeding layer existed but there were still zones transformation in the matrix. However, a clear bleeding layer can be observed in the visual bleeding test for the matrices with the same volume addition of fillers and $0.18 \%$ sbwp LS, see Figure 6 . This indicates differing dispersing effects on particles with the different water reducers.

Table 6 - The average dp/dt [Pa/s] of the mixtures at bottom and middle level after $2 \mathrm{~h}$.

\begin{tabular}{|c|l|c|c|}
\hline No. & \multicolumn{1}{|c|}{ matrix code } & average dp/dt in $2 \mathrm{~h}$ (bot.) & average dp/dt in $2 \mathrm{~h}$ (mid.) \\
\hline 1 & wc0.5wp0.5 & 0.250 & 0.116 \\
\hline 2 & wc0.7wp0.7 & 0.205 & 0.100 \\
\hline 3 & wc0.5wp0.5PCE0.18 & 0.168 & 0.068 \\
\hline 4 & ård-wc0.7wp0.585PCE0.18 & 0.151 & 0.077 \\
\hline 5 & ård-wc0.7wp0.5 & 0.152 & 0.066 \\
\hline 6 & ård-wc0.7wp0.5PCE0.18 & 0.164 & 0.091 \\
\hline 7 & ård-wc0.7wp0.5LS0.18 & 0.124 & 0.039 \\
\hline 8 & lim-wc0.7wp0.5 & 0.256 & 0.132 \\
\hline 9 & lim-wc0.7wp0.5PCE0.18 & 0.177 & 0.088 \\
\hline 10 & lim-wc0.7wp0.5LS0.18 & 0.115 & 0.024 \\
\hline
\end{tabular}

In Table 6, $d p / d t$ by HYPST measurements for all samples are compared, which indicates the combined effects of filler and water reducer on sedimentation. When the filler is applied as an additional powder, it generally stabilizes the cement paste due to the increased solid fraction and presumably also higher inter-particle forces, but the different fillers have different behaviour. The addition of the coarser Årdal filler prolongs the sedimentation period but gives a similar sedimentation rate, while the addition of the finer limestone may cause more flocculation and therefore induces a higher sedimentation rate. However, the additional limestone filler probably reduces the bleeding because the suspensions became more homogeneous and the sediment cake less compact. When the filler is applied as a replacement of cement to increase the $w / c$ ratio, e.g. Ardal-wc0.7wp0.5, lim-wc0.7wp0.5 and wc0.5wp0.5, the replacement with coarse Årdal filler lowered the sedimentation rate more visibly than the limestone filler did. This is possibly due to the greater zeta-potential change which Årdal filler induces for the particles in the matrix than limestone filler does. It is also likely that the flocculation state affected the bleeding. However, limestone filler decreases the total clear bleeding depth either with or without presence of water reducer. For the matrices with the addition of water reducer at a dosage of $0.18 \%$, the stabilizing effect of water reducer overshadows the effect from both types of fillers even at $40 \%$ addition or replacement. Both PCE and LS reduce the difference of the sedimentation performance shown for cement matrices without water reducer. The matrix with combined application of $L S$ and Årdal shows the lowest sedimentation rate in HYSPT measurements, which is probably due to the greatest zeta-potential change compared with that of the matrix without $L S$. 
Finally, it should be figured out that the particle interaction may vary with time and depth. The flocculation state of the particles would change with time and also be various at different zones of the mixtures. HYSPT experiments that carried out in $4 \mathrm{~h}$ take the effect of the particle flocculation on the sedimentation into account while zeta-potentials were measurement shortly after mixing. Therefore, the sedimentation rate may have varied at different levels in the mixtures, which may have induced different rates of expulsion of bleeding water. The matrix with fine limestone addition may therefore have had a lower sedimentation rate and bleeding rate at the bleeding front. However, as sedimentation continues, more serious flocculation could take place for finer particles than for the coarser ones with the same solid fraction, which results in a higher sedimentation rate.

\section{CONCLUSION}

The sedimentation and bleeding of cement matrices have been studied using HYSPT and bleeding measurements to investigate the effect of various combinations of fillers and water reducers on stability. To study how the interactions of the particles influence particle sinking, the zeta-potentials of cement and filler powder were measured electro-acoustically. It is found that the matrices show zones transformation, which coincides with the conceptual model previously proposed. To sum up:

1. The stability of a cement matrix with filler and water reducer cannot be characterized from final bleeding alone, because of turbid bleeding layers, particularly for PCE and filler.

2. Filler modifies the sedimentation and bleeding by varying the solid fraction, specific surface and inter-particle's forces.

3. Without water reducer, the addition of the coarser Årdal filler prolongs the sedimentation period but gives similar sedimentation rate, while the addition of the finer limestone filler induces higher sedimentation rate possibly due to the occurrence of more flocculation.

4. Filler can be used as either replacement or addition to cement. A finer filler does not always stabilize the matrix more than the coarser one. The replacement of coarse Årdal filler lowers the sedimentation rate more visibly than limestone, especially when combined with LS, probably due to the higher zeta-potential change it induces.

5. For the matrices with water reducer added at a solid dosage of $0.18 \%$, the stabilizing effect of water reducer overshadows the effect from both types of fillers even at $40 \%$ addition or replacement.

For further research, it is suggested to investigate the sedimentation, bleeding rate and bleeding of matrices with more types of fillers combined with water reducer and the effect of particle size, mineralogy and adsorption of admixtures on the stability of cement matrices.

\section{ACKNOWLEDGEMENT}

This research was part of research work of the COIN Centre led by SINTEF. Thanks to Espen Rudberg, previously at Mapei, for providing admixtures; to Norcem and Norstone for supplying cement, fillers and all PSD measurements as COIN partners; to G. Zirgulis for photographing and to the NTNU Concrete lab for their support with experiments. 
Nordic Concrete Research - Publ. No. NCR 58 - ISSUE 1 / 2018 - Article 7, pp. 107-125

\section{REFERENCES}

1. E. Mørtsell, M. Maage, S. Smeplass: "A Particle-Matrix Model for Prediction of Workability of Concrete”, Proceedings, International Conference on Production Methods and Workability of Fresh Concrete", Glasgow, Scotland 1995, 11 pp.

2. Josserand L, Coussy $\mathrm{O}$ and de Larrard F: "Bleeding of concrete as an ageing consolidation process”, Cement Concr. Res., V.36, 2006, pp.1603-1608.

3. Martius-Hammer T.A., Smeplass S., De Weerdt K., Peng Y.: "Stability of SCC-robustness for changes in water content and sand grading”, COIN Report, Trondheim, Norway, 2013, 27p

4. Peng Y., Jacobsen S.: "Influence of water cement ratio, admixtures and filler on sedimentation and bleeding of cement paste”, Cem. Conc. Res., V.54, 2013, pp. 133-142

5. Pedersen B.: "Alkali-reactive and inert fillers in concrete - Rheology of fresh mixtures and expansive reactions”, Doctoral thesis, NTNU, Trondheim, Norway, 2004, 292 pp.

6. Rhodes M., “Introduction to Particle Technology”, 2nd Edition, 2008, John Wiley \&Sons, Ltd, UK, ISBN 978-0-470-01428-8, 474 pp.

7. Stokes G.G., Math. Phys. Papers, 1901, Vol. 3

8. Richardson J F; Zaki W N.: "The sedimentation of a suspension of uniform spheres under conditions of viscous flow”, Chem. Eng. Sci., V.3, 1954, pp. 65-73

9. Kynch G. J:, “A theory of sedimentation”, Trans. Faraday Soc., V.48, 1952, pp.166-176

10. Powers T.C., “The Properties of Fresh Concrete”, Wiley \& Sons, London, 1968, 664 pp.

11. Steinour H. H.: "Further studies of the bleeding of Portland cement paste", Research Bulletin No. 4, Portland Cement Association, Skokie, USA, 1945

12. Zingg A., Winnefeld F., Holzer L., Pakusch J., Becker S., Gauckler L.: “Adsorption of polyelectrolytes and its influence on the rheology, zeta-potential, and microstructure of various cement and hydrate phases”, Journal of Coll. Inter. Sci., V.323, 2008, pp. 301-312

13. Zingg A., Winnefeld F., Holzer L., Pakusch J., Becker S., Figi R., Gauckler L.: "Interaction of polycarboxylate-based superwater reducers with cements containing different C3A amounts”, Cem. Conc. Comp., V.31, 2009, pp. 153-162

14. Hunter R.J.: "Zeta-potential in Colloid Science”. England: Academic Press, 1981

15. Williams R.A., Xie C.G., Bragg R., Amarasinghe W.P.K.: "Experimental techniques for monitoring sedimentation in optically opaque suspensions”, Coll. Surf., V.43, 1990, pp.1-32

16. Peng Y., Jacobsen S., De Weerdt K., Pedersen B:, "Model and test methods for stability of fresh cement paste”, ASTM-Adv. Civ. Engi. Mat., V.3, 2014, pp. 1-24, doi:10.1520 / ACEM20130097

17. Peng Y., "Sedimentation and bleeding of fresh cement paste": Doctoral thesis, Norwegian University of Science and Technology, Trondheim, Norway, 2014:89 ISBN 978-82-3260102-8 (printed), 2014:89 ISBN 978-82-326-0103-5 (electronic), 2014, NTNU, Norway

18. O'Brien R.W., Cannon D.W., Rowlands W.N.: "Electroacoustic Determination of Particle Size and Zeta-potential”, Journal of Coll. Inter. Sci., V.173, 1995, pp. 406-418

19. Lank J., C. Hirsch: "Impact of zeta-potential of early cement hydration phases on superwater reducer adsorption”, Cem. Conc. Res., Vol.. 37, 2007, pp. 537-542

20. Lesti M., Ng S., Plank J.: " $\mathrm{Ca}^{2+}$ Ion - Mediated Interaction between Microsilica and Polycarboxylate Comb Polymers in Model Cement Pore Solution", Journal of the Ame. Cer. Soc., V.93, 2010, pp. 3493 - 3498

21. Peng Y., Lauten R.A., Reknes K., Jacobsen S. "Bleeding and sedimentation of cement paste measured by hydrostatic pressure and Turbiscan”, Cem. Conc. Com., V.76, 2017, pp. 25-38. 